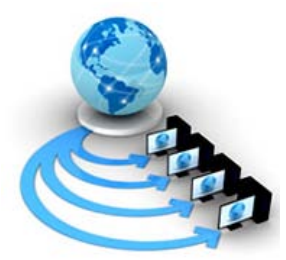

International Journal of Advanced Research in Computer Science

RESEARCH PAPER

\author{
Available Online at www.ijarcs.info
}

\title{
COMPARATIVE ANALYSIS OF HYBRID ROUTING PROTOCOLS ZRP, HCR AND ANTHOCNET
}

\author{
Harmandeep Kaur \\ M.Tech Student \\ Department of Computer Engineering \\ Punjabi University, Patiala, Punjab, India
}

\author{
Jasvir Singh \\ Assistant Professor \\ Department of Computer Engineering \\ Punjabi University, Patiala, Punjab, India
}

\begin{abstract}
Mobile ad hoc networks are data networks entirely made up of end user communication terminals (known as nodes). Each node in the network can act as an information sink (i.e. a receiver), a source and a router. All nodes have a transmission range, which is limited by their transmission power, attenuation and interference. Recently, a number of routing protocols have been designed to overcome limited bandwidth in the wireless medium, limited power supply, mobility and routing overhead. These protocols are classified into three different categories: Proactive Routing Protocols, Reactive Routing Protocols and Hybrid Routing Protocols. In this paper, we provide overview of hybrid routing protocols, Zone Routing Protocol (ZRP), Hybrid Cluster Routing (HCR) and AnthocNet. The comparison of Zone Routing Protocol (ZRP), Hybrid Cluster Routing (HCR) and AnthocNet are analysed on the basis of network performance parameters Throughput, Load, Packet Delivery Ratio using simulator OPNET 14.0
\end{abstract}

Keywords: - ZRP, HCR, AnthocNet, Hybrid Routing Protocol, MANET, Routing Protocols

\section{INTRODUCTION}

Wireless networks play a very prominent role in day to day communication. Wireless networks are of two types: Infrastructure Network \& Infrastructure less Network[1]. Infrastructure network contains fixed and wired gateways whereas Infrastructure less network contains multi hop wireless and it has no fixed infrastructure. Mobile ad hoc network is Infrastructure less Network. MANET is a selfconfiguring infrastructure less wireless network which consist mobile devices. Each mobile device is free to move anywhere independently in any direction[2]. So in this network, topology changes frequently as devices move independently and changes their links to other devices very quickly. Each mobile device act as a node in the network and that node forward the traffic to other nodes in the network, so each node act as a router. Routing protocols helps routers to find routes to send or receive packets to or from the network. Each router has the knowledge of the network to which it is directly attached. It is the routing protocol which first share this information with the immediate neighbors and then with all other routers throughout the network. Routing protocols sends or receives packets that contain routing information to or from the routers.

Routing protocols for mobile ad hoc network are broadly classified into three types[3]:

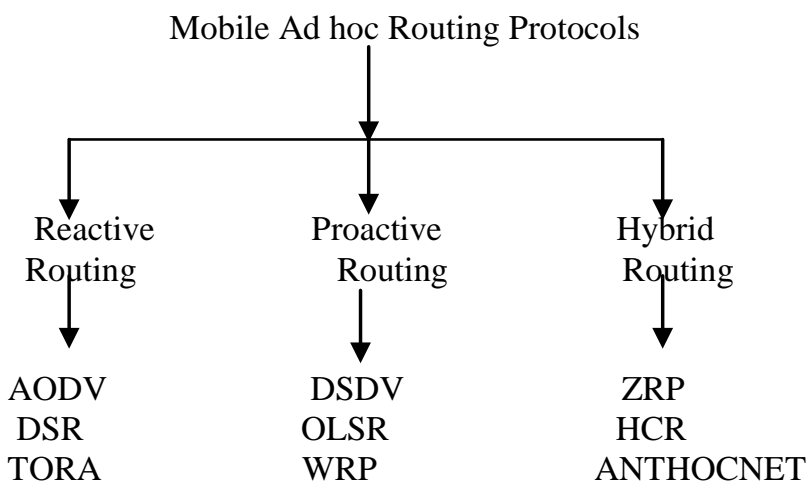

Figure1. Classification of MANET routing protocols

\section{A. Reactive or On-Demand Routing Protocols}

In Reactive Routing Protocols routes are not predefined. In this protocols, source node calls for route discovery phase to determine the route for packet transmission[4]. The route discovery mechanism is based on flooding algorithm which employs on technique that a node just broadcasts the packet to all its neighbors and intermediate nodes forwards the packets to their neighbors[1]. Overhead is smaller in reactive protocols but latency is higher.

\section{B. Proactive or Table-Driven Protocols}

In Proactive or Table-Driven, there are routing tables which contains the information of routes to all the nodes[2]. Routes are predefined in the routing tables and the packets are transferred to the routes[5]. As route is already specified in the table so packet forwarding is faster and as the routes have to be defined first before transferring the 
packets so overhead is more[6]. All routes are maintained at all the times so latency is low.

\section{Hybrid routing protocols}

It combines some of the characteristics of proactive protocols and some of the characteristics of Reactive protocols to gets better results. These protocols solve the routing overhead of proactive routing protocols and the delay of reactive routing protocols[3]. In this networks are separated into zones and suitable for large networks[1]. Hybrid routing protocols use reactive routing protocols for route discovery. It uses proactive routing protocols for table maintenance. Examples of Hybrid routing protocols which are used for route discovery are: ZRP, HCR and ANTHOCNET.

\section{OVerview of Zone Routing Protocol (ZRP)}

The ZRP[7] is hybrid reactive/proactive protocol. In ZRP proactive routing is inside the zone which is called the IntrAzone Routing Protocol (IARP) and outside the zone is reactive routing which is called the IntErzone Routing Protocol (IERP). The routing Zone is defined by the parameter called the zone radius. In these routing mechanism is different from standard flooding mechanism. The Interzone routing in ZRP is through a process known as bordercasting. Bordercasting is a packet delivery service that allows a node to send the packets to its peripherals nodes. This service is provided by component called the Bordercast Resolution Protocol (BRP). Fig. 2 illustrates[8] the routing with a routing zone of radius 2 hops. In this $S$ is the central node of the routing zone. The node which are 3 hops or more away from the central node are not come in routing zone because the routing zone of radius 2 hops. For example as shown in figure node $\mathrm{L}$ is outside the zone radius. The nodes which are exactly equal to zone radius are called the peripheral node. From figure it depicts that G, H, I, J and $\mathrm{K}$ are peripheral node. Nodes A to $\mathrm{K}$ are member of S's routing zone. The value 2 hops are not a physical distance it is node connectivity (hops).

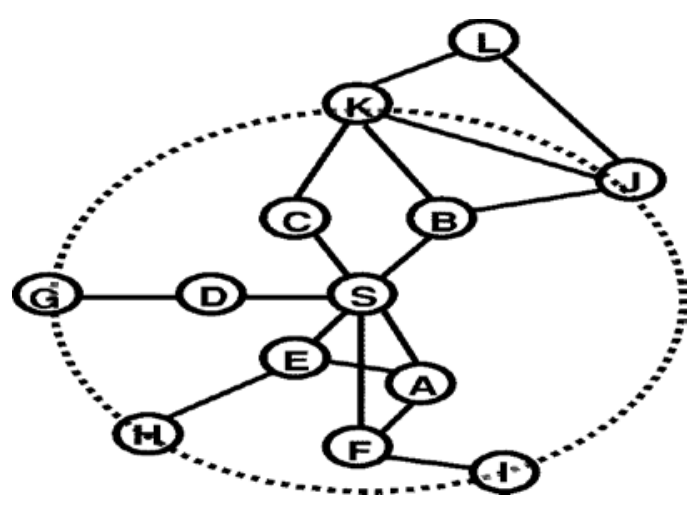

Figure2. A routing zone of radius 2 hops

\section{OVERVIEW OF HYBRID CluSTER ROUTING (HCR)}

Most of the cluster based routing protocols use proactive approaches within the cluster and reactive approach outside the cluster[9]. However this type of routing incurs overhead and delay. Therefore Hybrid Cluster based routing is designed which overcome the delay and routing overhead.
HCR protocol divides routing into two levels, i.e., intercluster routing and intra-cluster routing, respectively. Intercluster routing is on the higher level, which means a packet is routed cluster by cluster[10]. On the other hand, intracluster routing is on the lower level, which means a packet is routed within a cluster node by node. In HCR there are ordinary node, gateway node and cluster head.

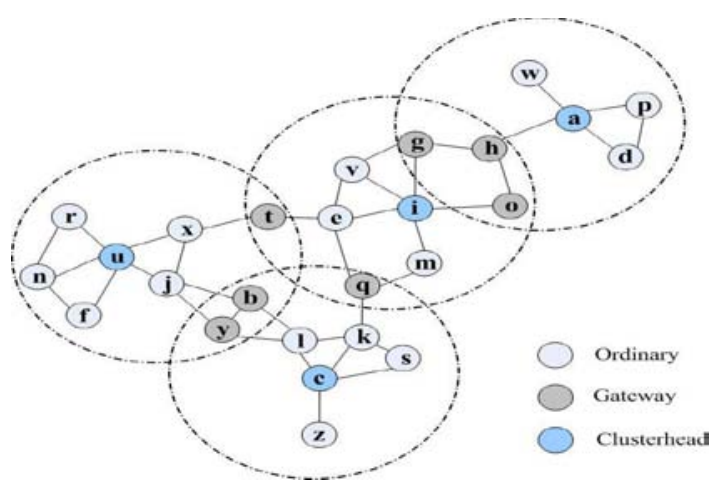

Figure3. An example of a 2-hop clustered network for HCR

\section{OVERVIEW OF ANTHOCNET}

Ant Colony Optimization has been inspired from the selfconfiguring and self-healing nature of social ant behavior[11].

In these when Ant move from one place to other they leave a substance called pheromone. These pheromone trails is followed by other ants. The ant which finds the shortest route is fastest to return and this ant reinforce the other ants by depositing food trail pheromone[12]. The other ants follow this route. In a computer field, the pheromone has been replaced by artificial stigmergy, the probabilities value used in the routing tables. To determine and update the probabilities values, intelligent agents are introduced to replace the real ants. There are two types of agents used one is the forward agents and other is the backward agents. In this when a source node as data to send to the destination, it sends ants like called the reactive forward ants. At destination they become backward ants. These update the routing table. The routing table $\mathrm{T}^{\mathrm{i}}$ in node $\mathrm{i}$ contains for each destination $\mathrm{d}$ and each possible next hop $\mathrm{n}$ a value $\mathcal{T}_{n d}^{i} \in \mathbb{R}$. $\mathcal{T}_{n d}^{i}$ is an estimate of the goodness of the path over $\mathrm{n}$ to $\mathrm{d}$, which we call pheromone. In this way, pheromone tables in different nodes indicate multiple paths between $\mathrm{s}$ and $\mathrm{d}$, and data packets can be routed from node to node as datagram. They are stochastically spread over the paths: in each node they select the next hop with a probability proportional to its pheromone value. Once paths are set up and the data session is running, s starts to send proactive forward ants to d[11]. These ants follow the pheromone values similarly to data packets. In this way they can monitor the quality of the paths in use. Moreover, they have a small probability of being broadcasted, so that they can also explore new paths. In case of link failures, nodes either try to locally repair paths, or send a warning to their neighbors such that these can update their routing table. 


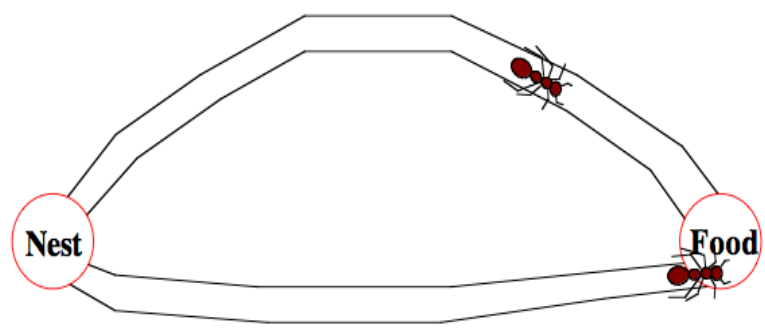

Figure4. ANTHOCNET Routing Protocol

\section{SIMULATION SETUP}

\section{A. Simulation Scenario}

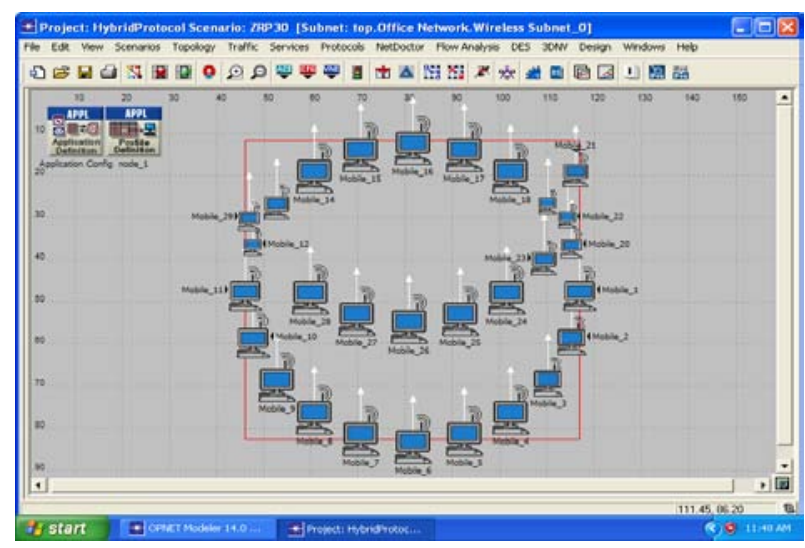

Figure5. Simulation Scenario of ZRP with 30 nodes To analyze the performance of ZRP[13], HCR[9] and ANTHOCNET[11] OPNET 14.0 simulator is used. Three different scenarios with 30, 40 and 50 node network are created for routing protocols ZRP, HCR and ANTHOCNET. The pause time, simulation time, simulation area, and Data rate are kept constant under all the scenarios. Simulation parameters used for the implementation of ZRP, HCR and ANTHOCNET are listed in the Table 1.

\section{B. Performance Metrics}

1) Throughput[14]:The high throughput describe the more packets are sent onto the ad hoc network and more will be the performance of the network. It is the average rate of successful message/ packets delivery over a communication channel[2]. The throughput is usually measured in bits per second (bit/s or bps), and sometimes in data packets per second or data packets per time slot. Throughput is used to measure the efficiency of the routing Protocol.

2) Packet delivery Ratio[15]: It is defined as the ratio of number of packets received by the destination node to the number of packets send by the source node. Mathematically it can be defined as $\mathrm{PDR}=\mathrm{S} 1 \div \mathrm{S} 2$, where $\mathrm{S} 1$ is the number of packets received by destination node and S2 is the number of packets send by the source node.

3) Load[16]: This statistics records the total amount of data submitted by the upper layer for transmission by the WLAN layer on all the nodes in the network. It is the number of packets sent to the network greater than the capacity of the network[5].
TABLE I SIMULATION PARAMETERS

\begin{tabular}{|c|c|}
\hline Parameters & Values \\
\hline Simulator & OPNET 14.0 \\
\hline Protocol Examined & ZRP,HCR and \\
\hline Simulation Time & 300 seconds \\
\hline Simulation Area & $100 \times 100$ \\
\hline Pause Time & .02 sec \\
\hline Buffer Size (bits) & Random way point \\
\hline Mobility Model & 256000 \\
\hline
\end{tabular}

\section{RESULTS \& DISCUSSION}

\section{A. Throughput}

From figure 6 depicts that average rate of successful message delivery over a communication channel in ZRP is more as compare to ANTHOCNET and HCR. The maximum value of throughput in ZRP is 2,513,952 bits/sec. It is also analyzed from figure that throughput in HCR and ANTHOCNET is same and less than that of ZRP. Hence, to send the data form source to destination ZRP is best in term of throughput.

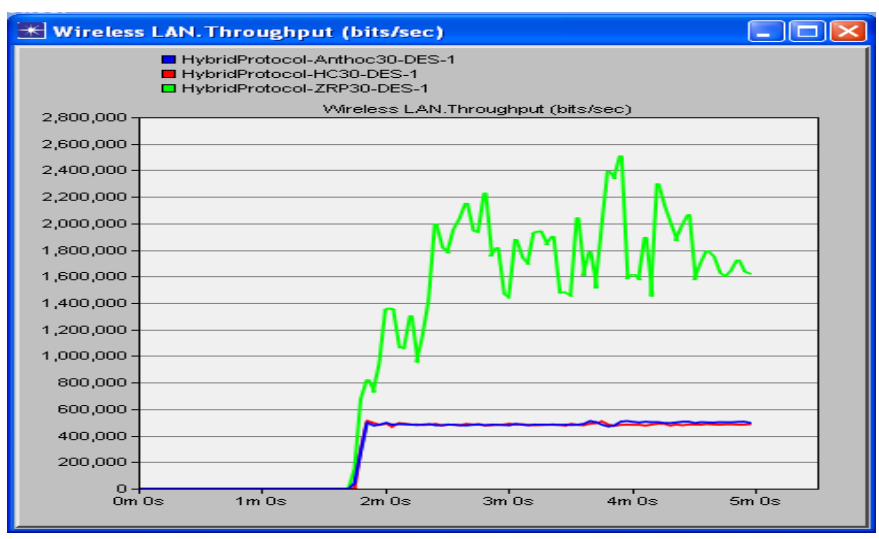

Figure6. Throughput comparison in routing protocols with 30 nodes 
It is shown in the figure 7 that throughput of ZRP is more than that of ANTHOCNET and HCR. The maximum value of throughput in ZRP is 4,692,475 bps. It is also analyzed from figure that throughput of HCR and ANTHOCNET are less than ZRP. Hence, to send the data form source to destination ZRP is superior in term of throughput at 40 node network.

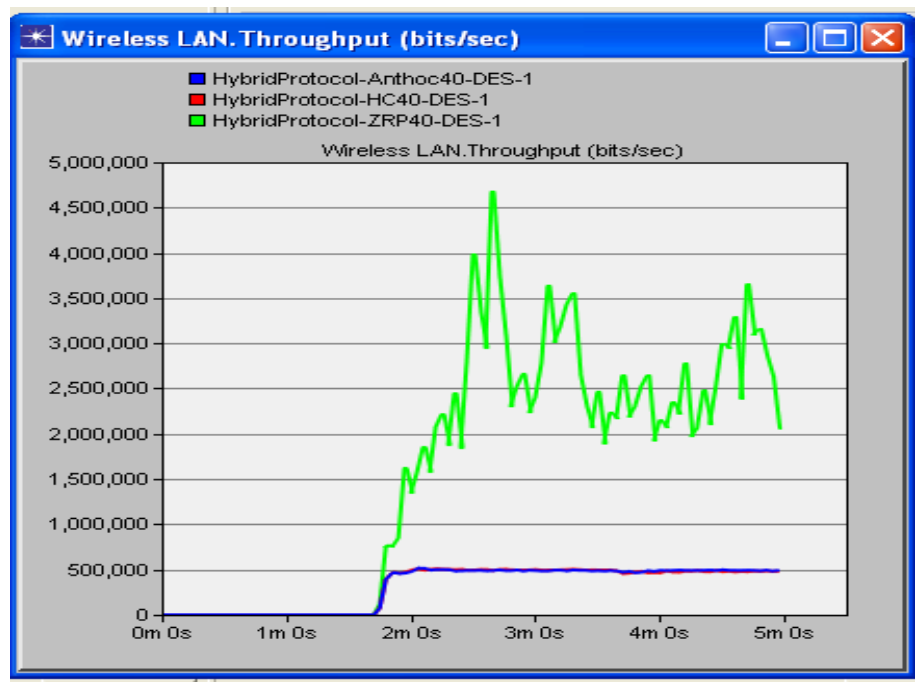

Figure7. Throughput comparison in routing protocols with 40 nodes

It is shown in the figure 8 that the throughput in ZRP is more as compare to ANTHOCNET and HCR. The maximum value of throughput in ZRP is 6,335,221 bps. It is depicts from result at 30, 40 and 50 node that throughput of ZRP is increased with increasing number of nodes. It is also depicts from figure that ZRP is best in terms of throughput at 50 node network.

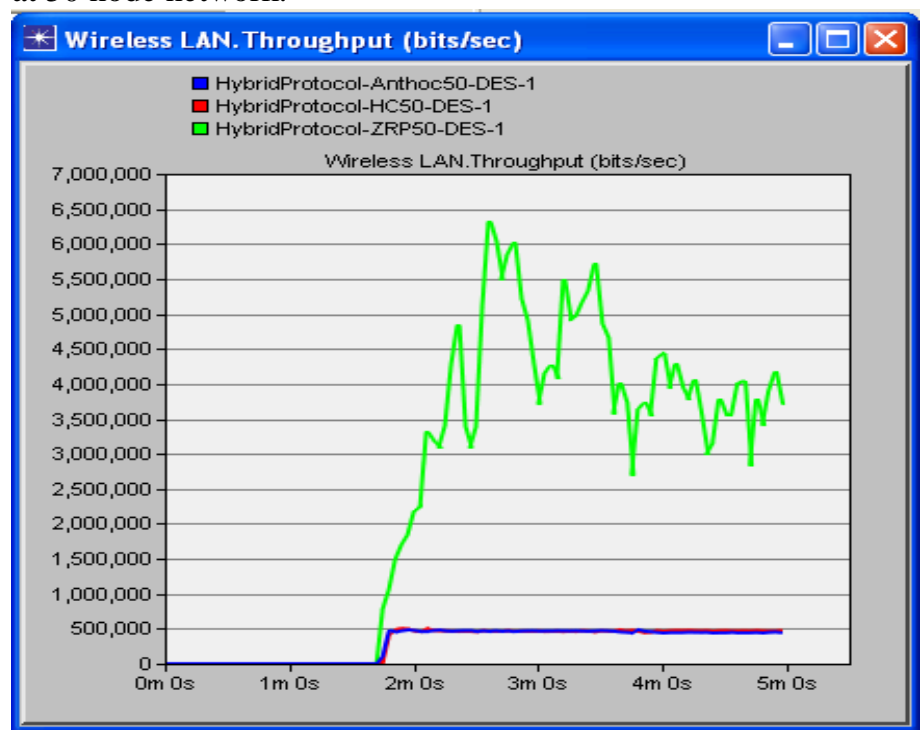

Figure8. Throughput comparison in routing protocols with 50 nodes

\section{B. Packet Delivery Ratio}

It is shown in the figure 9 that packet delivery ratio is more in ZRP as compare to HCR and ANTHOCNET. The maximum value of PDR in ZRP is 250. It is also analyzed from figure that packet delivery ratio in HCR is less as compare to ZRP and ANTHOCNET. Hence, ZRP is better in terms of packet delivery ratio.

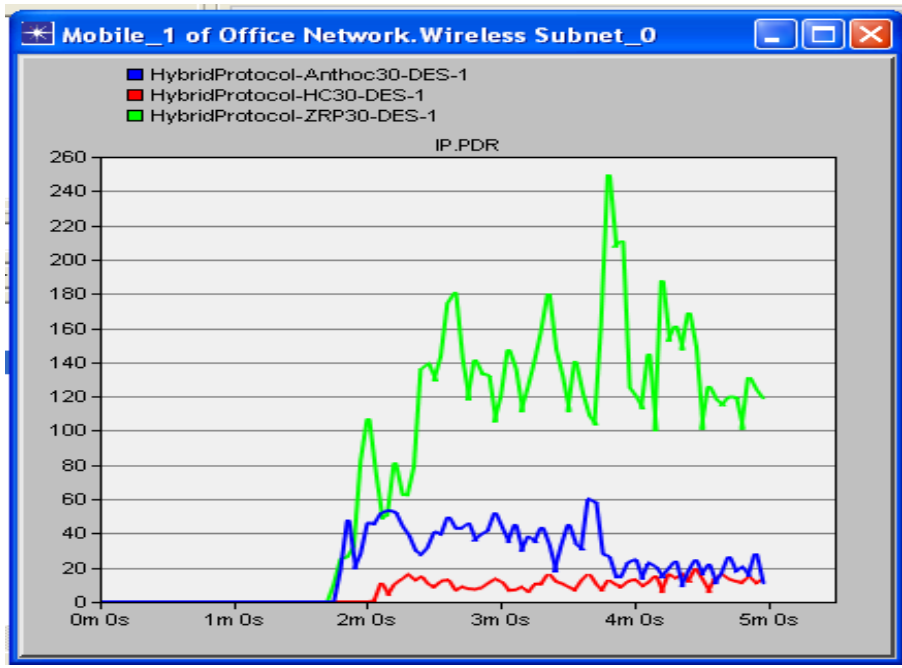

Figure9. PDR comparison in routing protocols with 30 nodes

It is shown in the figure 10 that packet delivery ratio is more in ZRP as compare to HCR and ANTHOCNET. It is also analyzed from figure that packet delivery ratio for HCR and ANTHOCNET is same but are less than ZRP. As shown in the figure that maximum packet delivery ratio in ZRP is 410. Hence, ZRP is superior in terms of packet delivery ratio.

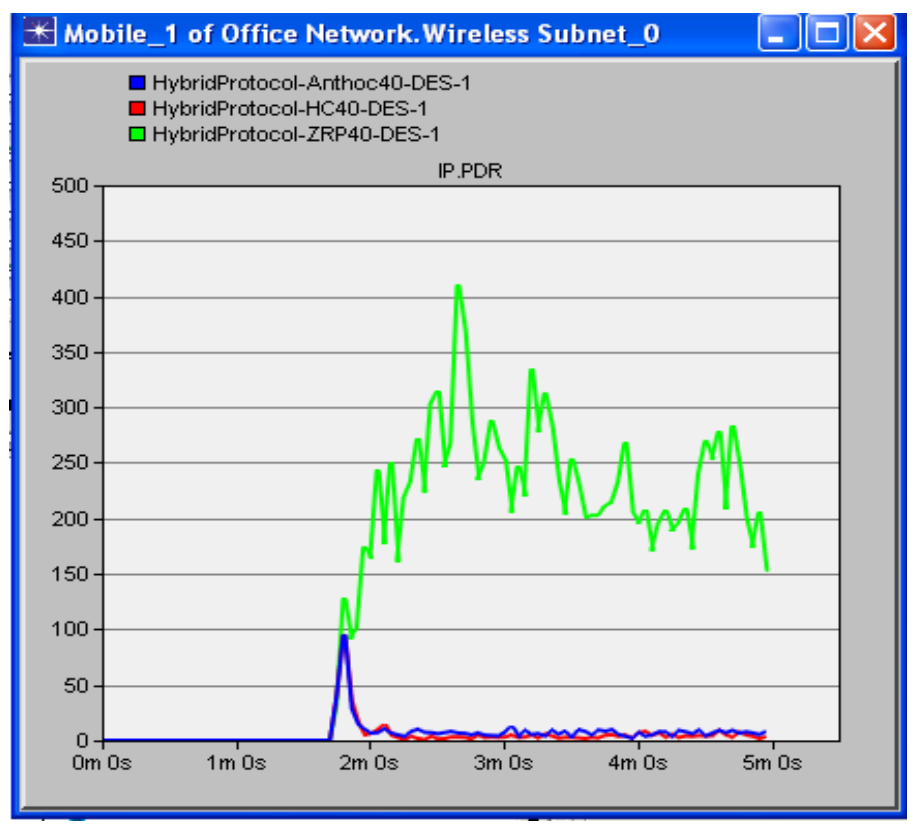

Figure10. PDR comparison in routing protocols with 40 nodes

It is shown in the figure 11 that ZRP packet delivery ratio in 50 node networks more than that of HCR and ANTHOCNET. The maximum PDR in ZRP is 330. It is also analyzed from figure that packet delivery ratio for 50 node network in ANTHOCNET is less than that of ZRP and HCR. The result shows that ZRP is better in terms of packet delivery ratio. 


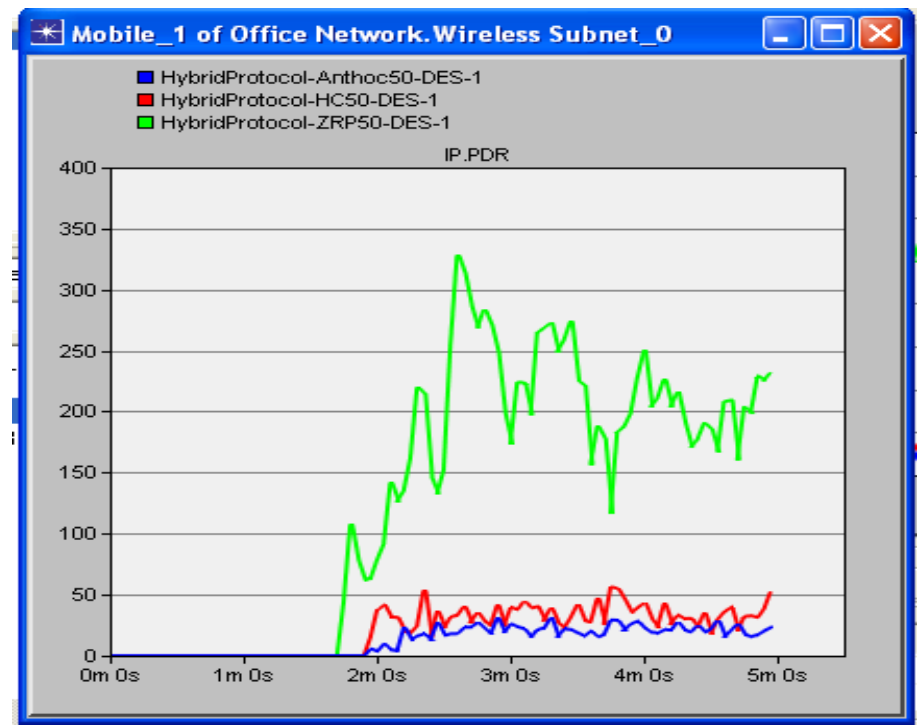

Figure11. PDR comparison in routing protocols with 50 nodes

\section{Load}

It is shown in the figure 12 that average Load over a network in ZRP is less as compare ANTHOCNET and HCR. The maximum value of load in ZRP is 2,352,168 bits/sec. It is also analyzed from figure that load in HCR is more as compare to ZRP and ANTHOCNET. Hence, to send the data form source to destination ZRP is best in term of Load at 30 node network.

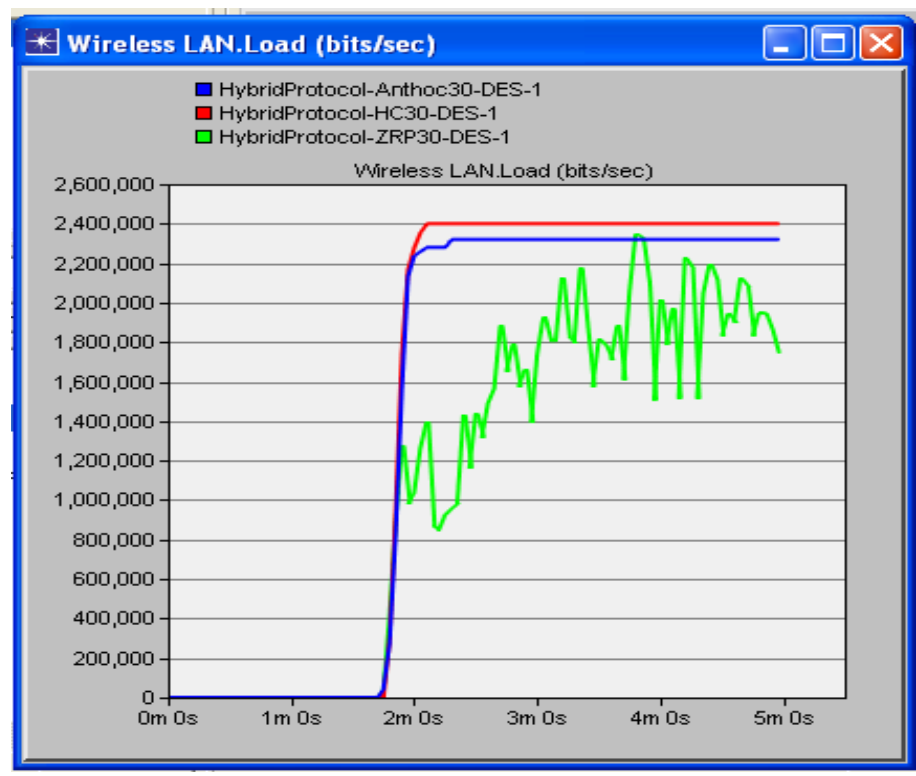

Figure12. Load comparison in routing protocols with 30 nodes

It is shown in the figure 13 that average Load over a network in ZRP is less as compare to ANTHOCNET and HCR. The maximum value of load in ZRP is $3,347,547$ bits/sec. It is also analyzed from figure that loads in ANTHOCNET and HCR is same but more than ZRP. The result shows that to send the data form source to destination ZRP is best in term of Load.

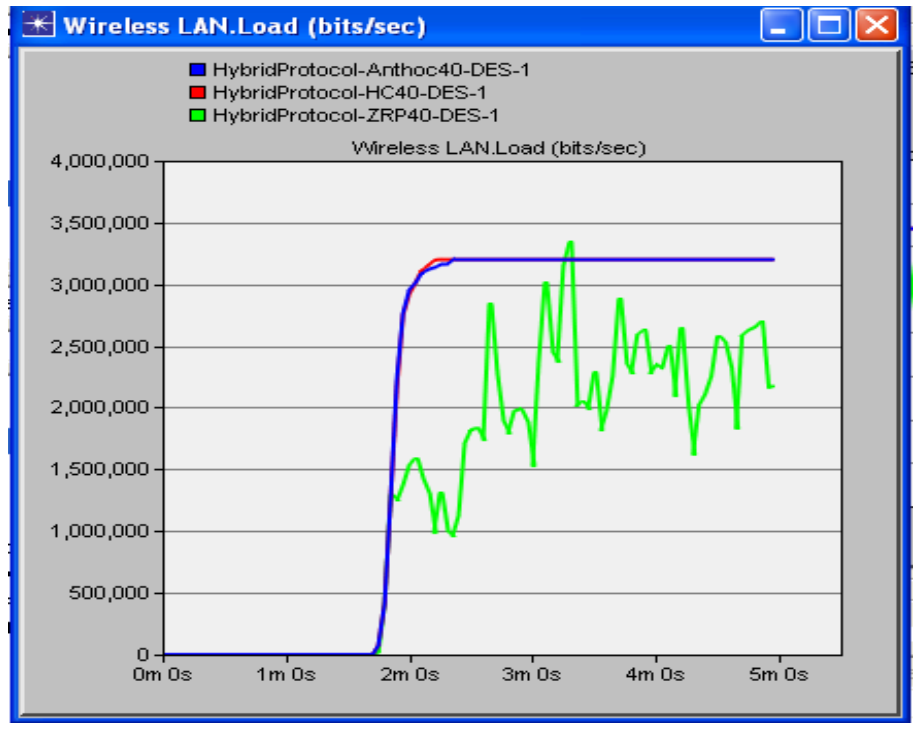

Figure13. Load comparison in routing protocols with 40 nodes

It is shown in the figure 14 that average Load over a network in ZRP is less as compare to ANTHOCNET and HCR. The maximum value of load in ZRP is 3,855,691 bits/sec. The results show that load is increased with increasing number of nodes. It is also analyzed from figure that load in ANTHOCNET is more as compare to ZRP and HCR. Hence, to send the data form source to destination over ZRP is better in term of Load.

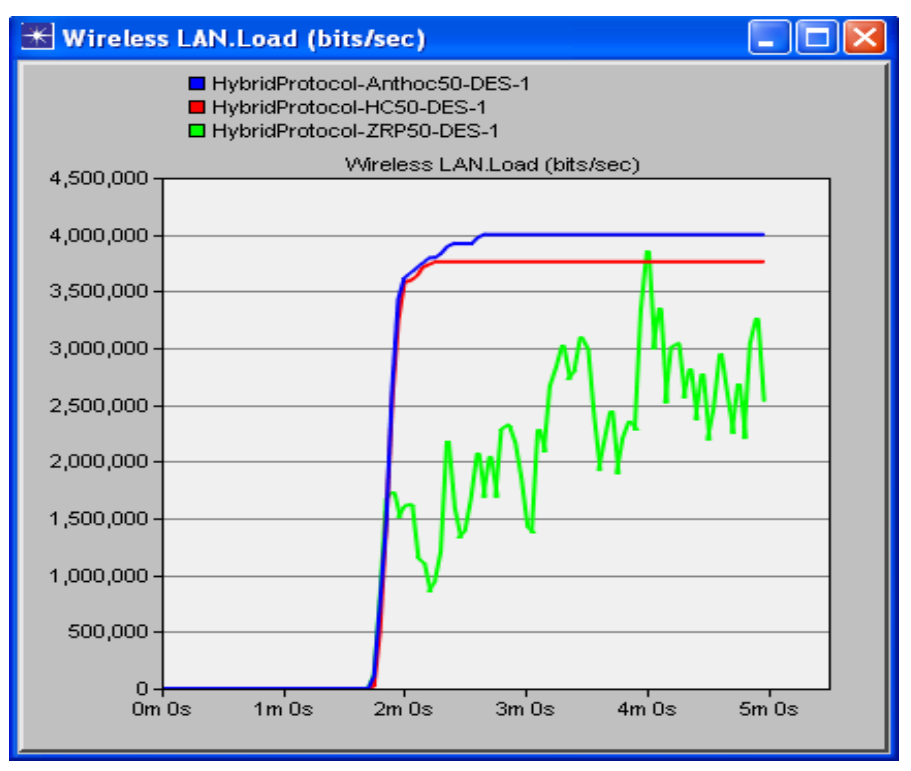

Figure14. Load comparison in routing protocols with 50 nodes

\section{CONCLUSIONS}

From the results, it is concluded that the network performance metrics, throughput, load and packet delivery ratio of ZRP are better than that of HCR and ANTHOCNET Routing Protocols. ZRP gives high throughput and packet delivery ratio than that of HCR and ANTHOCNET routing protocols at 30, 40 and 50 node network. The load in ZRP is less compare to HCR and ANTHOCNET. Hence, ZRP is superior to HCR and ANTHOCNET. 


\section{REFERENCES}

[1] H. Zhou, "A Survey on Routing Protocols in MANETs," Tech. Rep. MSU-CSE-03-08, no. 1, pp. 1-22, 2003.

[2] A. Kaur, “Amritpal Kaur," Int. J. Emerg. Trends Sci. Technol., vol. 2, no. 5, pp. 2491-2495, 2015.

[3] A. K. Gupta, H. Sadawarti, and A. K. Verma, "Review of Various Routing Protocols for MANETs,” Int. J. Inf. Electron. Eng., vol. 1, no. 3, pp. 251-259, 2011.

[4] L. Raja and S. Baboo, "Comparative study of reactive routing protocol (AODV, DSR, ABR and TORA) in MANET,” Int. J. Eng. Comput. Sci., vol. 2, no. 3, pp. 707-718, 2013.

[5] S. Kaur and S. Kaur, "Analysis of Zone Routing Protocol in MANET,” Int. J. Res. Eng. Technol., vol. 2, no. 9, pp. 520524, 2013.

[6] K. Gorantala, "Routing protocols in mobile ad-hoc networks," Master's Thesis Comput. Sci. June, 2006.

[7] Z. J. Haas, "A new routing protocol for the reconfigurable wireless networks,” vol. 2, pp. 562-566 vol.2, 1997.

[8] Z. J. Haas and M. R. Pearlman, "The performance of query control schemes for the zone routing protocol," IEEE/ACM Trans. Netw., vol. 9, no. 4, pp. 427-438, 2001.

[9] X. Niu, Z. Tao, G. Wu, C. Huang, and L. Cui, "Hybrid cluster routing: an efficient routing protocol for mobile ad hoc networks,” IEEE Int. Conf. Commun., vol. 8, no. c, pp. 35543559, 2006.
[10] I. No and G. Divya, “Analysis and Comparison of Various Clustering Techniques for Group Management in Mobile Ad Hoc Networks,” Int. J. Adv. Res. Comput. Sci. Res., vol. 3, no. 3, pp. 239-248, 2012.

[11] G. Caro, F. Ducatelle, and L. M. Gambardella, “AntHocNet $\square$ : an Ant-Based Hybrid Routing Algorithm for Mobile Ad Hoc Networks,” Parallel Probl. Solving from Nat. - PPSN VIII, 8th Int. Conf. LNCS 3242, no. August, pp. 461-470, 2004.

[12] T. S. and J. Singh, "Analysis of Ant Colony Optimization Based Protocols in MANETs,” Int. J. Adv. Res. Comput. Sci. Softw. Eng., vol. 4, no. 8, pp. 597-601, 2014.

[13] M. R. Pearlman and Z. J. Haas, "Determining the optimal configuration for the zone routing protocol," IEEE J. Sel. Areas Commun., vol. 17, no. 8, pp. 1395-1414, 1999.

[14] K. Sahadevaiah and O. B. Venkata Ramanaiah, "An Empirical Examination of Routing Protocols in Mobile Ad Hoc Networks,” Int. J. Commun. Netw. Syst. Sci., vol. 3, no. 6, pp. 511-522, 2010.

[15] P. Rohal, R. Dahiya, and P. Dahiya, "INTERNATIONAL JOURNAL FOR ADVANCE RESEARCH IN ENGINEERING AND TECHNOLOGY Study and Analysis of Throughput, Delay and Packet Delivery Ratio in MANET for Topology Based Routing Protocols ( AODV , DSR and DSDV )," vol. 1, no. Ii, pp. 54-58, 2013.

[16] A. S.Sethi and V. Y.Hnatyshin, The Practical OPNET User Guide for Computer Network Simulation. 2012. 